The Bayesian Approach to Audit Evidence:

Quantifying Statistical Evidence using the Bayes Factor

\author{
Koen Derks \\ Nyenrode Business University \\ K.Derks@nyenrode.nl \\ Jacques de Swart \\ Nyenrode Business University \& PwC Advisory \\ Jacques.de.swart@pwc.com \\ Eric-Jan Wagenmakers \\ University of Amsterdam \\ ej.wagenmakers@gmail.com \\ Ruud Wetzels \\ Nyenrode Business University \& PwC Advisory \\ ruud.wetzels@pwc.com
}

Running Head: The Bayesian Approach to Audit Evidence 


\title{
The Bayesian Approach to Audit Evidence: \\ Quantifying Statistical Evidence using the Bayes Factor
}

\begin{abstract}
Statistical methods play an important role in auditors' analyses of auditee's data. A key aspect in the statistical approach to auditing is assessing the strength of evidence for or against a hypothesis. Unfortunately, the frequentist statistical methods often used by auditors in practice cannot provide the statistical evidence that audit standards advocate directly nor easily. In this article we discuss an alternative approach that can provide this evidence: Bayesian inference. Firstly, we explore the philosophical differences between frequentist and Bayesian inference. Secondly, we discuss misconceptions in the interpretation of frequentist statistical evidence, and finally we discuss how Bayesian inference allows the auditor to obtain and interpret statistical evidence in line with audit standards via its alternative to the frequentist $p$ value, the Bayes factor. We contribute to audit theory and practice by showing how Bayesian inference can quantify audit evidence.
\end{abstract}

Keywords: audit evidence; analytical procedures; Bayes factor; substantive testing.

\section{INTRODUCTION}

Auditors play a key role in preserving the integrity of the financial reporting of companies, (non-profit) organizations, and governments. The objective of the auditor is to provide stakeholders of the auditee with an opinion with reasonable assurance about the completeness and fairness of the assertions as presented by the auditee's management in the financial statements (ISA 200; International Auditing and Assurance Board (IAASB), 2018; 
AU-C 200, American Institute of Certified Public Accountants (AICPA), 2021; AS 1001, Public Company Oversight Board (PCAOB), 2020). Auditing standards provide guidance on how to obtain this reasonable assurance. These audit standards mandate that the auditor's opinion must be based on persuasive (rather than conclusive) audit evidence consisting of information that can support or contradict management's assertions in the financial statements (ISA 500 paragraph 5c, International Auditing and Assurance Board (IAASB), 2018; AU-C 500 paragraph 6, American Institute of Certified Public Accountants (AICPA), 2021; AS 1105 paragraph 2, Public Company Accounting Oversight Board (PCAOB), 2020). Unfortunately, the frequentist statistical methods that are standard in practice (Stewart, 2012) cannot provide the statistical evidence that audit standards advocate directly nor easily.

In this article we introduce an alternative approach that can quantify this evidence in line with auditing standards: Bayesian inference. As an alternative to the frequentist statistical approach to auditing, the Bayesian approach primarily focuses on quantifying evidence, and it has therefore been advocated as a more suitable statistical framework for audit practice (Johnstone, 2018; Stewart, 2013). In the Bayesian approach to auditing, the auditor quantifies statistical evidence using the Bayes factor (Kass \& Raftery, 1995). Because the Bayes factor can provide the statistical evidence that the audit standards advocate, we believe that it has the potential to enhance the way that auditors currently analyze and evaluate statistical evidence from a sample. While not the first mention of the Bayes factor in an audit context (see Johnstone, 2018, pp. 33-34), the main contributions of this article are to explain how the Bayes factor enables the auditor to intuitively quantify audit evidence, to demonstrate its advantages over standard frequentist methodology, to show how it can be applied in practice using a variety of relevant examples, and to enable practical use by supplying easy-to-use, free, and open-source software to calculate the Bayes factor for a variety of audit scenarios. 
Two types of audit evidence can be distinguished: non-statistical and statistical. Nonstatistical audit evidence is collected from supervision, inquiry, or correspondence with the auditee (Bennett \& Hatfield, 2013; Perry, 2011; Yin, 2020). Statistical audit evidence is collected from statistical procedures and analyses performed by the auditor (Gillet \& Srivastava, 2000; van den Acker, 2000), such as testing a subset of relevant control systems to determine whether or not they meet quality requirements (Li, Raman, Sun, \& Yang, 2020), performing analytical procedures to assess relationships between financial and non-financial data in the auditee's databases (Appelbaum, Kogan, \& Vasarhelyi, 2018; Daroca \& Holder, 1985), or performing audit sampling to obtain reasonable assurance that auditee's financial statements are free of material misstatement (AICPA 2019; Dowling \& Leech, 2007). Regardless of the type of statistical procedure or analysis, the auditor requires a data sample to perform statistical inference on a certain characteristic of the auditee.

Where statistical sampling is applied, probability theory is required (ISA 530, paragraph 5g, International Auditing and Assurance Board (IAASB), 2018). There are two main schools of probability theory: frequentist and Bayesian (Wagenmakers et al., 2008). In current practice, frequentism is the dominant methodology to analyze data and arrive at statistical audit evidence. Audit guides implicitly nudge auditors towards a frequentist hypothesis testing framework to, for example, evaluate their samples using confidence intervals or $p$ values (AICPA, 2019; Stewart, 2012). However, frequentism has several wellknown drawbacks. Most importantly, a frequentist hypothesis test does not give the auditor what the audit standards advocate: statistical evidence that can support or contradict the auditor's conclusions. In particular its main decision-making tool, the $p$ value, is not a measure of statistical evidence, it strictly provides only indirect support against the conclusions made by the auditor. Moreover, the frequentist $p$ value is ineffective at quantifying evidence that can support the auditor's conclusions, and it is inefficient since 
monitoring evidence as the data come in and stop gathering data when a certain evidential threshold is reached (e.g., when the $p$ value is smaller than 0.05 ) is not allowed (Wagenmakers, 2007; van Batenburg, 2018). For these reasons, many scholars have raised concerns about the efficiency, transparency, and applicability of the frequentist methodology to audit practice (Beck et al., 1985; Hubbard \& Lindsay, 2008; Johnstone, 1986; Johnstone, 1990; Kim, Ahmed, \& Ji, 2018; Kinney, 1975; Scott, 1973). In Section 3 of this article, we will go into more detail about the concerns of frequentist hypothesis testing when it comes to auditors' effectiveness and efficiency.

On the other hand, in this article we show that Bayesian hypothesis testing using the Bayes factor as a measure of audit evidence fits the auditor well because it can quantify evidence for and against hypotheses (Wagenmakers, 2007), it allows for sequential adding of information (Rouder, 2014), and Bayesian analyses allow for incorporation of expert knowledge or other existing information into the statistical analysis (Corless, 1972). In a Bayesian approach the auditor samples data with the aim of obtaining relative evidence for two competing hypotheses. The rationale is that there is evidence for the null hypothesis over the alternative hypothesis if the data are more likely to occur under the null hypothesis (and vice versa). Because a Bayesian approach considers the likelihood of the data under two competing hypotheses, it is possible to directly compare the relative evidence for the hypotheses. This allows auditors to obtain statistical evidence that can support or contradict their conclusions, something that is often desired but impossible using a frequentist or nonstatistical approach. Both the frequentist and Bayesian approaches facilitate attribute sampling, monetary unit sampling, estimation using confidence or credible intervals, and most importantly in an audit context- statistical hypothesis testing. Nonetheless, the two approaches differ in their approach to hypothesis testing and therefore in how the resulting statistical evidence can be interpreted. In Section 4, we demonstrate the differences in 
interpretation between the statistical evidence resulting from both approaches in a variety of audit scenarios. We show that the Bayes factor enables auditors to intuitively quantify statistical audit evidence.

A key aspect of Bayesian inference is that the auditor needs to specify a so-called prior distribution. The prior distribution represents the information about an unknown parameter of interest to the auditor, which in turn is combined with the probability distribution of new data (e.g., the likelihood) to yield the posterior distribution. This updating of information from the prior to the posterior distribution forms the basis of any Bayesian inference. Even though the audit is inherently a continuous process (Leslie, 1984), and audit evidence is considered “cumulative in nature” (ISA 200, paragraph A30, International Auditing and Assurance Board (IAASB), 2018; AU-C 500 paragraph A3, American Institute of Certified Public Accountants (AICPA), 2021), the use of the Bayesian philosophy in audit practice is scarce. This may, in part, be explained by the fact that literature discussing the role of Bayesian philosophy in the quantification of audit evidence is scarce. This is unfortunate since the Bayesian philosophy has several properties that fit well with the audit process. In Section 3 we describe in detail how Bayesian hypothesis testing using the Bayes factor addresses the concerns of frequentist hypothesis testing when it comes to auditors' effectiveness and efficiency.

The structure of this article is as follows. We will first provide a theoretical introduction into statistical audit evidence. Next, we discuss and compare statistical audit evidence from a frequentist and Bayesian point of view. We show that -in contrast to the frequentist $p$ value- the Bayes factor can quantify evidence that supports or contradicts the auditor's conclusions regarding management's assertions in the financial statements. In the section thereafter we reason that the $p$ value limits the auditor in their activities and that the Bayes factor does not suffer from these limitations. Finally, to illustrate how the auditor can 
quantify evidence from a wide range of activities, we use the Bayes factor in a reanalysis of four typical audit questions. The last section presents our concluding comments.

\section{TWO APPROACHES TO STATISTICAL AUDIT EVIDENCE}

Audit evidence is the subject of auditing standards ISA 500 (IAASB, international firms), AU-C 500 (AICPA, private firms) and AS 1105 (PCAOB, public firms). IAASB standard ISA 500 describes audit evidence as "[i]nformation used by the auditor in arriving at the conclusions on which the auditor's opinion is based" (paragraph 5c) and states that audit evidence "comprises both information that supports and corroborates management's assertions, and any information that contradicts such assertions" (paragraph A.1;

International Auditing and Assurance Board (IAASB), 2018). Both AICPA standard AU-C 500 (paragraph 6) and PCAOB standard AS 1105 (paragraph 2) describe audit evidence in a similar manner. Clearly, auditing standards advocate that audit evidence should be able to support or contradict the auditor's conclusions about management's assertions in the financial statements.

For example, to obtain audit evidence to support or contradict the hypothesis that the auditee's recorded financial transactions do not contain misstatement that exceeds materiality, an auditor can inspect a subset of all financial transactions for their accuracy. However, because the auditor only inspects a subset of the population a statistical hypothesis test is needed to assess the level in which the evaluated subset of data supports or contradicts the auditor's hypothesis about all the data. To make concrete how the auditor can engage in statistical hypothesis testing, we will focus on a specific activity in the audit where statistical inference is common: audit sampling. However, statistical evidence can be obtained from 
many types of audit activities and, as we will show in Section 4, the differences between the frequentist and Bayesian approach are equally relevant there.

\subsection{Hypothesis testing in audit sampling}

Audit sampling enables the auditor to obtain evidence with respect to a specific hypothesis about the misstatement in the population from which items are selected (International Auditing and Assurance Board (IAASB), 2018). Because the auditor only inspects a sample of the population, the hypothesis cannot be evaluated with absolute certainty. However, because the auditor is required to obtain a reasonable assurance they must evaluate the hypothesis to a level of certainty, and therefore it needs to be clear how much information is required to reach this level. The audit standards prescribe that the information from a sample is sufficient when it has reduced the sampling risk to an acceptably low level (ISA 530, paragraph 5c, International Auditing and Assurance Board (IAASB), 2018; AU-C 530, paragraph 5, American Institute of Certified Public Accountants (AICPA), 2021). There are two types of sampling risk that can lead to an incorrect conclusion about the financial misstatements (Elliott \& Rogers, 1972). First, there is $\alpha$ : the risk of incorrectly deciding that the population contains material misstatement when in fact it does not (i.e., a Type-I error). Second, there is $\beta$ : the risk of incorrectly deciding that the population does not contain material misstatement when in fact it does (i.e., a Type-II error). According to the audit standards, auditors are primarily concerned with reducing the second type of risk, $\beta$, since it affects effectiveness and their ability to provide an appropriate audit opinion.

If the auditor engages in statistical audit sampling, the audit standards state that they can quantify the sampling risk (ISA 530, paragraph 5g (IAASB, 2018); AU-C 530, paragraph .05 (AICPA, 2021), AU 350, paragraph .46 (PCAOB, 2020)). However, when doing so the auditor is free to choose which philosophy of probability is applied: a frequentist or a 
Bayesian philosophy. To illustrate the differences between these two philosophies, we will evaluate a running example of an hypothesis test along the lines of both philosophies.

Suppose that an auditor is required to assess the financial statements of a publicly traded company. In this example, the auditor must determine if no more than three percent of the auditee's purchase orders contain an incorrect signature. The auditor's one-sided null hypothesis stating that the misstatement in the purchase orders does not exceed the maximum tolerable error rate of three percent can be formulated as $H_{0}: \theta \leq 0.03$, whereas the one-sided alternative hypothesis, which is the opposite of the null hypothesis, reads $H_{1}: \theta>0.03$. In these hypotheses, $\theta$ represents the proportion of purchase orders with an incorrect signature. A different way of testing these hypotheses is to include the value of the maximum tolerable error rate in the alternative hypothesis, see the appendix to this article for further details.

However, since these purchase orders have not been subject to an audit before the true value of $\theta$ is unknown. Therefore, the auditor would like to decide about the credibility of the hypothesis $H_{0}$ by selecting several purchase orders and determining the correctness of the signature. Suppose that, after inspecting a sample of 99 purchase orders from the auditee's archives, the auditor finds that none of these orders contain an incorrect signature.

\subsection{Frequentist null hypothesis testing}

The traditional method of analyzing these sample outcomes to arrive at a conclusion about $\theta$ is frequentist null hypothesis significance testing (NHST) (AICPA, 2019; Elliott \& Rogers, 1972; Stewart, 2012). In NHST, the auditor formalizes a so-called null hypothesis that represents the minimal value of $\theta$ at which their alternative hypothesis is not supported (Fisher, 1934), which in our example is the hypothesis $H_{0}: \theta \leq 0.03$. Unfortunately, NHST only allows for quantifying evidence against the null hypothesis $H_{0}$. This means that the auditor is unable to quantify evidence that can support the null hypothesis and, furthermore, is 
unable to quantify evidence that supports the alternative hypothesis if an alternative hypothesis is defined.

When testing the null hypothesis, the auditor assumes that $H_{0}$ is true and sets out to gather data to evaluate $H_{0}$. The rationale behind NHST is that increasingly stronger evidence will be obtained against $H_{0}$ (the population is free of material misstatement) when the data become increasingly implausible assuming the truth of $H_{0}$. If sufficient evidence is obtained that contradicts $H_{0}$, it can be rejected with reasonable assurance. NHST allows the auditor to quantify the evidence against $H_{0}$ using the $p$ value, which expresses the probability of seeing the observed sample outcome or more extreme -but unobserved-sample outcomes, assuming the truth of $H_{0}$. Hence, the frequentist $p$ value enables the auditor to assess the risk of incorrectly rejecting the null hypothesis, which in this case is the $\alpha$ risk. However, it is also possible to derive the $\beta$ risk. To explain how these two risks can be assessed in NHST, we will first show to calculate the $\alpha$ risk and subsequently show the calculation of the $\beta$ risk.

To perform the statistical inference, the data from the sample needs to be connected to the null hypothesis about the population parameter by means of a probability distribution (Lehman \& Romano, 2006). For example, if the auditor assumes that the purchase orders in the sample are all independent observations and that the only parameter that exerts influence on the sample outcomes is the probability of misstatement $\theta$, a binomial distribution can be applied (Johnstone, 1990; Sorensen, 1969) ${ }^{1}$, see Equation 1.

$$
\text { Probability of } k \text { errors in } n \text { items }=\left(\begin{array}{l}
n \\
k
\end{array}\right) \theta^{k}(1-\theta)^{n-k}
$$

To determine when $H_{0}: \theta \leq 0.03$ should be rejected for a given sample size, the auditor must calculate the maximum number of incorrect signatures that can be observed

\footnotetext{
${ }^{1}$ AICPA (2019) prescribes the binomial distribution for attribute sampling and the Poisson distribution for monetary unit sampling. However, the binomial distribution can also be used for monetary unit sampling.
} 
while the risk of incorrectly rejecting the null hypothesis is still sufficiently low. Suppose that the auditor has determined $\alpha$-the risk of incorrectly deciding that the population contains material misstatement when in fact it does not- to be five percent. This threshold is referred to as the significance level. In this case, the rule for rejection of $H_{0}$ is $k \geq 7$ because if it is true that $\theta=0.03$, then the probability of finding 7 or more incorrect signatures in the sample of 99 purchase orders equals $2.98 \%$ (see Equation 1). This probability is lower than the required significance level of 5 percent. This procedure for rejecting $H_{0}$ can also be regarded as rejecting the null hypothesis if the $p$ value associated with the observed value of $k$ is less than or equal to the significance level $\alpha$.

To continue our running example, we will calculate the $p$ value. In this case, calculating the $p$ value is trivial because the data is certain to occur under the null hypothesis. However, we will show the calculation here anyway. The $p$ value is the probability of finding $k=0$ to $k=99$ incorrect signatures in the sample, given that the purchase orders contain three percent misstatement, and equals $p=1$ (Equation 2). This means that, given the truth of the null hypothesis, the data from the sample are certain to occur. Since the calculated $p$ value of 1 is higher than the significance level $\alpha=0.05$, the auditor cannot reject the null hypothesis.

$$
p=\sum_{k=0}^{k=99}\left(\begin{array}{c}
99 \\
k
\end{array}\right) 0.03^{k}(1-0.03)^{99-k}=1
$$

A default conclusion for a $p$ value larger than the significance level $\alpha$ is to not reject and thus maintain- the null hypothesis $H_{0}: \theta \leq 0.03$. However, the interpretation of a larger $p$ value as support for the null hypothesis is flawed (Goodman, 2008). The fallacy in this statement is best described through the words "absence of evidence is not evidence of absence” (Altman \& Bland, 1995, p. 485; Keysers, Gazzola, \& Wagenmakers, 2020). Simply put, the finding that data contain no evidence against the null hypothesis does not imply that they contain evidence that supports the null hypothesis. Since the $p$ value is solely a measure 
of evidence against the null hypothesis, it fails to address the extent to which the sample provides support for the null hypothesis. Thus, based on the $p$ value, auditors are unable to quantify statistical evidence that can support their conclusion that the misstatement in the population does not exceed the maximum tolerable error rate.

On the other hand, a $p$ value lower than the significance level $\alpha$ generally leads the auditor to reject the null hypothesis $H_{0}: \theta \leq 0.03$, and to accept the alternative hypothesis $H_{1}: \theta>0.03$. However, as we have discussed, in NHST the $p$ value only concerns the null hypothesis $H_{0}$ and not an alternative hypothesis. Therefore, if the auditor uses this procedure to substantiate their conclusion that the sample supports the opposing hypothesis, $H_{1}$, they fall into a statistical trap (Berger \& Sellke, 1987; Berkson, 1942; Wagenmakers, 2007). The (im)plausibility of the alternative hypothesis is not considered in the computation of the $p$ value, and since the computation of the $p$ value is solely based on the evaluation of the data in light of the null hypothesis it provides only an indirect argument for the alternative hypothesis. Thus, the $p$ value fails to address the extent to which the sample supports the alternative hypothesis $H_{1}$. Based on the $p$ value, the auditor is unable to quantify statistical evidence that can support the conclusion that the misstatement in the population exceeds the maximum tolerable error rate. This also implies that auditors are unable to statistically contradict the conclusion that the misstatement in the population does not exceed the maximum tolerable error rate.

As mentioned in the auditing standards, the auditor can also calculate the sampling risk $\beta$-the risk of deciding that the population does not contain material misstatement when in fact it does. In order to calculate $\beta$ for an alternative point hypothesis about the population misstatement, the auditor needs to make an assumption about $\theta$. Suppose the auditor assumes that the population misstatement is equal to $\theta=0.04$, which is slightly higher than the maximum tolerable error rate. When assuming the truth of this hypothesis, the risk of failing 
to reject the null hypothesis $H_{0}: \theta \leq 0.03$ is the probability of finding an outcome that would yield a $p$ value above five percent (e.g., $k=7$ misstatements would give $p<.05$ and thus lead to rejection of $H_{0}$ ). Hence, the sampling risk $\beta$ can be calculated as the probability of finding $k=0$ to $k=6$ incorrect signatures in the sample under the binomial $(k \mid n=99, \theta=0.04)$ distribution and equals $\beta=0.90$. This calculation shows that, if the population truly contains misstatement slightly higher than the maximum tolerable error rate, there is a 90 percent chance to incorrectly decide that the population does not contain material misstatements. The reason that this is high is because the assumed population material misstatement is very close to the upper bound of the null hypothesis. The sampling risk $\beta$ would be lower if the sample size would be higher, or if the assumed population material misstatement would be higher.

Since the auditing standards state that audit evidence consists of information that can support or contradict the auditor's conclusions, the inability of the $p$ value to provide support for the null- and alternative hypothesis makes it arguably unsuited for quantifying statistical evidence in an audit context. Unfortunately, this is not limited to audit sampling but applies to any (analytical or substantive) procedure in which the auditor quantifies statistical audit evidence using the $p$ value. However, in the next section we show that, by using a Bayesian hypothesis test, the auditor can quantify the required statistical evidence via the Bayes factor.

\subsection{Bayesian hypothesis testing}

In contrast to NHST, where the auditor's evidence is solely based on the model for the null hypothesis $H_{0}$, a Bayesian hypothesis test incorporates both hypotheses $H_{1}: \theta>0.03$ and $H_{0}: \theta \leq 0.03$ into the statistical procedure. The driving force behind Bayesian inference is Bayes' theorem (Jeffreys, 1939), which stipulates how existing information about an event $A$ can be updated using information from a new event $B$ (Equation 3).

$$
p(A \mid B)=p(A) \times \frac{p(B \mid A)}{p(B)}
$$


On a conceptual level, Bayes' theorem embodies a fundamental principle in the audit: the notion that audit evidence is "cumulative in nature" (ISA 200, paragraph A.30, International Auditing and Assurance Board (IAASB), 2018, AU-C 500, paragraph A3, American Institute of Certified Public Accountants (AICPA), 2021) and that the auditor can therefore aggregate audit evidence over the audit.

In line with accumulating evidence, it is important to state the current level of information before an analysis is performed. Hence, in the Bayesian framework, a prior distribution needs to be defined for every aspect of the statistical model that is to be estimated. The prior distribution is a probability distribution that represents the auditor's existing information about the possible values of parameters or hypotheses. In audit sampling, the prior distribution can be used to incorporate existing evidence about the possible values of the misstatement $\theta$ into the sampling procedure (Corless, 1972). For example, the auditor's risk assessments on the inherent risk and control risk are information that can be incorporated into the prior distribution (Derks et al., 2021; Stewart, 2013).

To use Bayes' theorem for hypothesis testing, the auditor must also first quantify their existing evidence about the plausibility of the two competing hypotheses using so-called prior probabilities. The prior probability $\mathrm{p}\left(H_{0}\right)$ incorporates the auditor's existing evidence about the probability of the null hypothesis $H_{0}$ occurring before seeing any information from a data sample. Vice versa, the prior probability $\mathrm{p}\left(H_{1}\right)$ incorporates the auditor's existing evidence about the probability of the alternative hypothesis $H_{1}$. This makes it possible that information for or against a given hypothesis is evaluated and incorporated prior to choosing the sample to test. The ratio of prior probabilities is called the prior odds and is an indication of the relative plausibility of the hypotheses before analyzing the intended sample. 
When performing an audit, new information $y$ from a sample is observed and the auditor aims to update the prior probability $\mathrm{p}\left(H_{i}\right)$ of the hypothesis $H_{i}$ to a posterior probability $\mathrm{p}\left(H_{i} \mid y\right)$. This is done via Bayes' rule, which allows the auditor to update their prior knowledge about the hypothesis $\mathrm{p}\left(H_{i}\right)$ with the evidence that is contained in the data for or against this hypothesis $\mathrm{p}\left(y \mid H_{i}\right)$, resulting in the posterior probability of this hypothesis (see Equation 4). Given the cumulative nature of audit evidence described in the audit standards, this philosophy of revising and aggregating evidence is more in line with audit practice than that of NHST.

$$
\underbrace{p\left(H_{i} \mid y\right)}_{\text {Posterior probability }}=\underbrace{p\left(H_{i}\right)}_{\text {Prior probability }} \times \underbrace{\frac{p\left(y \mid H_{i}\right)}{p(y)}}_{\text {Evidence }}
$$

The posterior probability $\mathrm{p}\left(H_{i} \mid y\right)$ represents the probability that the hypothesis $H_{i}$ is true, conditioned on the existing (prior) information and the evidence from the sample. For example, a posterior probability $\mathrm{p}\left(H_{0} \mid y\right)=0.95$ implies that, given the existing audit evidence and the evidence in the sample, there is a 95 percent probability of correctly deciding that $H_{0}$ is true. Hence, the posterior probabilities can be intuitively related to the sampling risks $\alpha$ and $\beta$. More concretely, when accepting $H_{0}$, the posterior probability $\mathrm{p}\left(H_{1} \mid\right.$ y) can be interpreted as the $\beta$ risk. Vice versa, when rejecting $H_{0}$ and accepting $H_{1}$, the posterior probability $\mathrm{p}\left(H_{0} \mid y\right)$ can be interpreted as the $\alpha$ risk.

However, because the auditor is interested in comparing the evidence for two hypotheses, they can employ Bayes' theorem to obtain the ratio of posterior probabilities for $H_{0}$ and $H_{1}$, the posterior odds. The posterior odds can be denoted as the product of the prior odds and the relative evidence for the hypotheses (Equation 5).

$$
\underbrace{\frac{p\left(H_{0} \mid y\right)}{p\left(H_{1} \mid y\right)}}_{\text {Posterior odds }}=\underbrace{\frac{p\left(H_{0}\right)}{p\left(H_{1}\right)}}_{\text {Prior odds }} \times \underbrace{\frac{p\left(y \mid H_{0}\right)}{p\left(y \mid H_{1}\right)}}_{\text {Relative evidence }}
$$


Since the posterior odds depend on the prior odds as well as the information from the sample, and because it can be very difficult to define the prior odds, it is common practice to quantify the relative evidence that the sample contains using the ratio of evidence for the two hypotheses. This ratio is called the Bayes factor, and it quantifies the change in prior to posterior odds brought about by the data (Kass \& Raftery, 1995). Compared to the $p$ value, the Bayes factor is a direct comparison of the evidence for both hypotheses on the sample (Equation 6).

$$
B F_{01}(y)=\frac{p\left(y \mid H_{0}\right)}{p\left(y \mid H_{1}\right)}
$$

Since the Bayes factor is a ratio, it can quantify evidence in both directions. It is this specific property of the Bayes factor that fits well with the audit standards' description of audit evidence because it enables the auditor to quantify evidence that can support their hypotheses as well as evidence that can contradict their hypotheses. For example, a Bayes factor in favor of $H_{0}$ of $7\left(B F_{01}=7\right)$ indicates that the sample outcomes are 7 times more likely to occur under the null hypothesis $H_{0}$ than under the alternative hypothesis $H_{1}$. Furthermore, because $B F_{10}=\frac{1}{B F_{01}}=\frac{1}{7}, B F_{01}=7$ also indicates that the sample outcomes are 7 times less likely to occur under the alternative hypothesis $H_{1}$ than under the null hypothesis $H_{0}$. Because of the ease of interpretation of the Bayes factor, it is rapidly being adopted in many areas of business and science such as Psychology (Heck et al., 2020; Ly, Verhagen, \& Wagenmakers, 2016), Sociology (Bollen et al., 2012; Lynch \& Barlett, 2019), and Economy (Cipriani, Constantini, \& Guarino, 2012; Richard \& Vecer, 2021). Furthermore, Bayes factor calculations have been made very easy in many standard situations such as the (partial) correlation test (Wetzels \& Wagenmakers, 2012), the t-test (Rouder et al., 2009; Wetzels et al., 2009), or the ANOVA (Rouder et al., 2012; Wetzels, Grasman, \& Wagenmakers, 2012) and implementation in easyto-use software such as JASP (JASP Team, 2021; Love et al., 2019). 
In a similar fashion to the $p$ value there exist only subjective decision rules for what Bayes factor represents sufficient evidence since what may be considered convincing evidence in a low-risk audit might not be considered as convincing evidence in a high-risk audit. However, to aid practitioners in interpreting the Bayes factor, a collection of labels has been proposed and reiterated in numerous academic articles (Jeffreys, 1961; Wetzels et al., 2011; van Doorn et al., 2020). Table 1 displays these evidential thresholds, which auditors can use to interpret the strength of evidence provided by the Bayes factor in practice. Because the Bayes factor is a ratio $B F_{01}=7$ implies moderate evidence in favor of the null hypothesis and at the same time implies moderate evidence against the alternative hypothesis. In similar fashion, $B F_{01}=20$ implies strong evidence in favor of the null hypothesis and at the same time strong evidence against the alternative hypothesis. Note that, although $p$ values and Bayes factors mostly agree about which hypothesis is supported by the data, they often disagree about the strength of this support (Wetzels et al., 2011).

To continue our running example, a Bayesian auditor starts their audit sampling procedure by first specifying a prior probability distribution $\mathrm{p}(\theta)$ that reflects their existing information about the parameter $\theta$ : the misstatement in the population. The two hypotheses $H_{0}: \theta \leq 0.03$ and $H_{1}: \theta>0.03$ are defined as the range of the prior distribution that corresponds to the hypotheses' restrictions with respect to $\theta$. This means that the prior probability for the hypothesis $H_{0}$ corresponds to the total probability under the prior distribution on $\theta$ in the range $[0 ; 0.03]$. Vice versa, the prior probability for the hypothesis $H_{1}$ corresponds to the total probability under the prior distribution on $\theta$ in the range $(0.03 ; 1]$. To be able to compute the probability under both hypotheses the auditor needs to define the prior probability distribution that fits this situation.

If the auditor has assessed inherent risk and internal control risk according to the Audit Risk Model, they can incorporate this information into the prior distribution. For illustrative 
purposes it is convenient to specify a uniform beta $(1,1)$ prior distribution that represents negligible information about the misstatement $\theta$ (Stewart, 2013). The auditor has assessed both inherent risk and internal control risk as "medium" which, according to their audit guide, translates into a reduction in the sample size of $\Delta n=33$. These unseen samples are assumed to be correct and can be incorporated in the prior distribution by setting the $\beta$ parameter of the prior distribution to $1+\Delta n=34$ (Derks et al., 2021; Steele, 1992). For the beta $(\alpha=1, \beta=34)$ distribution, the prior odds in favor of the hypothesis $H_{0}$ are $\frac{0.645}{0.355}=1.817$, see Figure 1 .

After seeing the information from a sample of $n$ items of which $k$ contain an error, the prior distribution is updated by the binomial likelihood to a posterior distribution $\mathrm{p}(\theta \mid n, k)$ according to Bayes' theorem (Equation 7).

$$
\underbrace{p(\theta \mid n, k)}_{\text {Posterior }}=\underbrace{p(\theta)}_{\text {Prior }} \times \frac{p(k \mid n, \theta)}{\underbrace{p(y=k, n)}_{\text {Evidence }}}
$$

Similar to the prior distribution, the posterior distribution induces a probability for the occurrence of the hypotheses. The posterior probability for the hypothesis $H_{0}$ corresponds to the probability mass assigned by the posterior distribution to the values of $\theta$ in the range [0; 0.03). Vice versa, the posterior probability for the hypothesis $H_{1}$ corresponds to the probability mass assigned by the posterior distribution to the values of $\theta$ in the range $[0.03$; $1]$.

After being updated by the sample of $n=99$ items of which $k=0$ contain an error, the posterior distribution is the beta $(1+0=1,34+99=133)$ distribution. The posterior odds in favor of $H_{0}$ induced by the posterior distribution are therefore $\frac{0.983}{0.017}=57.824$, see Figure 1 . The posterior probability $\mathrm{p}\left(H_{0} \mid y\right)=0.983$ implies that there is a 98.3 percent probability that the population does not contain misstatements that exceed the maximum tolerable error rate. This means that, when accepting $H_{0}$, there is a 1.7 percent probability that the auditor 
incorrectly judges that the population is free of material misstatement. This probability is sufficiently low to find the statement in the null hypothesis credible. Vice versa, this also implies that there is a 98.3 percent probability that the auditor correctly judges that the population is not materially misstated.

Because we know the prior odds and the posterior odds, we can calculate the Bayes factor by dividing the two. Thus, the Bayes factor in this example can be calculated as $B F_{01}=$

$\frac{57.824}{1.817} \approx 31$, which implies that the data are about 31 times more likely to occur under $H_{0}$ than under $H_{1}$. This Bayes factor implies very strong evidence in favor of $H_{0}$ (see Table 1 ).

\subsection{Comparison of frequentist and Bayesian conclusions}

Note that a frequentist analysis $(p=1>\alpha)$ only facilitates a statement about the (im)plausibility of the data (or data more extreme) under the hypothesis $H_{0}$, and forces the auditor to conclude that the null hypothesis cannot be rejected. As mentioned in the previous section, using this $p$ value the auditor cannot say that there is evidence in favor of the null hypothesis. The Bayes factor differs from the $p$ value in that it can quantify evidence directly in favor of the null hypothesis and that it provides an intuitive interpretation of this evidence. That is, the Bayes factor $B F_{01} \approx 31$ shows that $H_{0}$ is many times more likely than $H_{1}$ and that there is strong evidence in favor of the null hypothesis that the misstatement in the population does not exceed the maximum tolerable error rate. Moreover, this calculation shows that, because in a Bayesian procedure the auditor considers both a null and an alternative hypothesis, they can intuitively assess both the $\alpha$ and $\beta$ risk.

In sum, because the Bayes factor can quantify audit evidence in both directions it is more in line with the philosophy of evidence described in the audit standards than the $p$ value. However, the Bayes factor is not only an attractive alternative to the $p$ value because of its intuitive theoretical interpretation, it also removes some of the practical limitations that the $p$ 
value brings. In the next section we describe these limitations of the $p$ value in more detail and explain why the Bayes factor does not suffer from these limitations. In Section 4 we will discuss three practical examples of Bayes factor calculations in an audit context.

\section{PRACTICAL IMPLICATIONS}

In this section we illustrate that the use of frequentist NHST using the $p$ value limits auditors in their effectiveness and in their efficiency in quantifying audit evidence. Next, we show that the Bayes factor does not suffer from these limitations and that it is therefore an attractive alternative to the $p$ value. To illustrate which improvements the Bayes factor brings, we focus on two properties of the $p$ value that are undesirable: the $p$ value cannot provide evidence for the null hypothesis, and it does not allow for sequential sampling (Wasserstein \& Lazar, 2016; Rouder, 2014; Wagenmakers, 2007, Wagenmakers, Gronau \& Vandekerckhove, 2019). We explain why the Bayes factor does not suffer from these limitations and what this implies for the auditor in practice.

\subsection{The $p$ value cannot provide evidence for the null hypothesis}

The $p$ value can lead to an ineffective audit when the auditor wishes to support the null hypothesis, but due to the nature of frequentist hypothesis testing they are unable to obtain statistical evidence that quantifies this support. This limits the auditor because it takes away many possibilities for statistical analyses in which a null hypothesis is the focus of the investigation. The Bayes factor does not suffer from this limitation because it allows the auditor to quantify evidence in both directions, thereby enabling the auditor to obtain evidence for the null hypothesis if desired.

One can think of many scenarios (other than the sampling scenario in the previous section) where supporting the null hypothesis is the main goal of the statistical analysis, as 
doing so can contribute to the existing evidential matter in the audit. Suppose that the auditor wants to support the null hypothesis that an auditee's inventory is valued fairly, wants to confirm the auditee's accounts receivable or wants to confirm the auditee's sales transactions. As we have discussed in Section 2, relying on the $p$ value makes supporting this null hypothesis impossible. This can be appreciated by noting that $p=.35$ will not be strong evidence for the null hypothesis when the sample size is small (i.e., absence of evidence), but the same $p$ value will be strong evidence for the same hypothesis when the sample size is large (i.e., evidence of absence). To illustrate, a sample of 14 items containing 1 misstatement and a sample of 284 items containing 10 misstatements both yield $p=.35$. Hence, it is not possible to gather evidence supporting the null hypothesis based on the $p$ value. In Section 4 we discuss two more examples in which the auditor wants to support the null hypothesis: a situation in which the auditor wants to support the conclusion that the data in the auditee's financial statements are subject to Benford's law (example 2) and a situation where an auditor of a tax authority wants to support the conclusion that all taxable persons are treated equally (example 4). We show in more detail how in these commonly occurring scenarios the $p$ value does not fit well with the audit question at hand because it is unable to quantify support for the null hypothesis.

However, by reporting a Bayes factor the auditor can quantify evidence directly in favor of the null hypothesis, thereby removing this limitation and providing a more fitting answer to their question. In a Bayesian approach the auditor can make a statement about how much more likely the data are under the null hypothesis versus the alternative hypothesis. This makes it possible to directly compare the evidence in the data for both hypotheses, thus allowing the auditor to support or contradict their conclusion. Hence, the Bayesian approach to audit evidence fits well with the audit question at hand because supporting a null 
hypothesis can contribute just as much to the existing evidential matter as rejecting a null hypothesis.

\subsection{The $p$ value does not allow for sequential sampling}

The $p$ value can lead to an inefficient audit when the auditor already has enough evidence to support a particular hypothesis, but due to the nature of frequentist hypothesis testing they still need to perform the remainder of the planned work. This limits the auditor in their efficiency because at this point, they are performing more work than necessary. The Bayes factor does not suffer from this limitation since it allows the auditor to monitor the evidence for any hypothesis while the data come in (Rouder, 2014). Having access to such information during the audit increases efficiency for the auditor because it allows them to modify sampling procedures at an early stage when necessary, or to stop sampling procedures when sufficient evidence is obtained.

In a frequentist analysis using the $p$ value, the auditor must complete data collection before analyzing the sample results (Berger \& Wolpert, 1988, Lindley, 1993). That is because, given the sampling risk $\alpha$, each time the auditor looks at the intermediate results there is an $\alpha$ percent chance that a significant $p$ value is produced when the null hypothesis is true. As a result, the probability of incorrectly rejecting the null hypothesis increases as a function of the number of times that the auditor looks at the results (Armitage, McPherson, \& Rowe, 1969; Wagenmakers, 2007). Thus, to maintain control over this type of sampling risk in the frequentist framework, the auditor must finish their intended sampling plan before analyzing the sample. In contrast, because a Bayesian analysis is not dependent on a sampling plan, the auditor is allowed to monitor the evidence for a particular hypothesis and to stop data collection when enough evidence is obtained (Wagenmakers, Gronau \& Vandekerckhove, 2019). From a Bayesian point of view "It is entirely appropriate to collect 
data until a point has been proven or disproven, or until the data collector runs out of time, money, or patience." (Edwards, Lindman, \& Savage, 1963, p. 193).

To illustrate the benefits of sequential sampling, let's consider the following example. Suppose that the auditor wants to obtain evidence to support the assertion that a certain population contains misstatements lower than a certain threshold $t$. Statistically speaking, they can then define the null hypothesis as: $H_{0}: \theta \geq t$. In this case, the auditor wants to sample until they can reject the null hypothesis. They have planned a sample size such that - when no misstatements are found- they can reject the null hypothesis that the population contains material misstatement with a sampling risk $\alpha$ of five percent. As it turns out, the sample contains a single misstatement, which means that the auditor cannot reject this null hypothesis. If the auditor still wants to be able to reject the null hypothesis using the $p$ value, they will need to plan an extension for their sample. Because there is an increase in the sampling risk $\alpha$ after looking at the data, one possible way to proceed is to plan a follow-up sample in which they adjust the maximum $p$ value. However, this practice generally results in a substantial extension of the sample. To make this concrete, in most audit guides it is prescribed that the auditor inspects at least an additional number of items equal to the initial sample (AICPA, 2019, Appendix B). For this case, that would imply an increase in the sample size from $n=99$ to at least $n=198$.

In contrast to the frequentist analysis using the $p$ value, in the Bayesian framework the auditor is allowed to build upon the information from the previous sample without penalty. Therefore, they can coherently extend their sample from $n=99$ to $n=156$ (the sample size that they would have got when initially planning for one misstatement in the sample), or to any other $n$ depending on the desired strength of evidence (i.e., the desired Bayes factor). Other than being more efficient in terms of the sample size, this Bayesian sample size 
extension is arguably more intuitive and easier to explain for the auditor than a frequentist one.

\section{APPLYING THE BAYES FACTOR IN A MODERN AUDIT}

To facilitate the use of the Bayes factor and illustrate the benefits of a Bayesian approach to audit evidence, we will now apply the Bayes factor to three typical audit questions. Please see the online appendix to this article for details about the derivations of various statistics and the calculations of the Bayes factors in this section. As a first example, we will apply digit analysis using Benford's law to a financial data set. In the second example, we will analyze historical data from an auditee's sales revenue to uncover evidence that supports a potential seasonal effect. In the final example, we analyze an auditee's classification algorithm to investigate to what degree the data reflect algorithmic fairness.

\subsection{Example 1: Assessing Benford's law}

Benford's law (Benford, 1938) has been advocated as a simple, (arguably) effective method for auditors to not only identify discrepancies in data, but to uncover potential data manipulation in financial statements (Durtschi, Hillison, \& Pacini, 2004), ERP systems (Ma'arif et al., 2020), or official information released by authorities (Wei \& Vellwock, 2020). Simply put, Benford's law states that in many naturally occurring collections of numbers the leading digit is likely to be small. More concretely, a set of numbers is said to satisfy Benford's law if the leading digit $d \in\{1, \ldots, 9\}$ occurs with probability:

$$
\mathrm{p}(d)=\log _{10}\left(1+\frac{1}{d}\right)
$$

Benford's law is, among other applications, mentioned as an analytical procedure in an early stage of the audit (Nigrini \& Mittenmaier, 1997). For example, small deviations from 
Benford's law may suggest that the data have passed a reasonableness test, while large deviations may be a sign of possible data manipulation or the need for further investigation (Drake \& Nigrini, 2000). The goal of the analysis in this example is to determine how much evidence the data provide for the statement that the leading digits in the recorded values of a population of items follow Benford's law.

\subsubsection{Data}

The data for this example come from the financial statements of the Sino Forest Corporation's 2010 Report (Nigrini, 2012). For illustrative purposes, we will only analyze the leading digits of the recorded values but this procedure can be generalized to include the first two, or last, digits. The frequencies of the leading digits in the sample are displayed in Table 2.

\subsubsection{Frequentist analysis}

In the NHST framework, the auditor wants to test the null hypothesis $H_{0}$ that the first digits are distributed according to Benford's law. An example application of this procedure is described by Varma and Khan (2012), who used Benford's law to identify potential fraud in a similar population. The hypothesis $H_{0}: p_{d}=\log _{10}\left(1+\frac{1}{d}\right)$ is assessed by means of the $p$ value.

Using a Chi-squared test $\left(X^{2}=7.652, d f=8\right)$, the $p$ value for these data is 0.468 . The interpretation of this $p$ value is: assuming that the first digits are distributed according to Benford's law, there is a 46.8 percent probability that the auditor would have found the observed (or more extremely deviating) distribution of first digits in the data set. In a standard fashion, the conclusion would be to not reject, and thus maintain, the null hypothesis $H_{0}$.

\subsubsection{Bayesian analysis}


In a Bayesian analysis of Benford's Law (Good, 1967; Sarafoglou et al., 2020), the null hypothesis is compared, by means of the Bayes factor, against the alternative hypothesis which states that the first digits are not distributed according to Benford's law (i.e., the digit probabilities are free to vary). The prior probabilities for the hypotheses are set to be equal: $\mathrm{p}\left(H_{0}\right)=\mathrm{p}\left(H_{1}\right)=0.5$. The prior distribution for the alternative hypothesis is assumed to be a $\operatorname{Dirichlet}\left(\alpha_{1}, \alpha_{2}, \ldots, \alpha_{9}\right)$ distribution with all $\alpha$ parameters set to 1 . Note that, in this case, a parameter $\alpha_{d}$ of the Dirichlet distribution reflects the prior count for the digit $d$ and can be adjusted to incorporate prior information into the alternative hypothesis.

The corresponding Bayes factor in favor of the null hypothesis is $B F_{01}=6899678$, which implies that the data are 6899678 times more likely (extreme evidence) to have occurred under the hypothesis that the first digits are distributed according to Benford's law than under the hypothesis that they are not. Because the prior probabilities are set to be equal, the Bayes factor equals the posterior odds, which implies that the posterior probability for the null hypothesis can be deduced as $\mathrm{p}\left(H_{0} \mid y\right)=\frac{B F_{01} \times p\left(H_{0}\right)}{B F_{01} \times p\left(H_{0}\right)+\left(1-p\left(H_{0}\right)\right)}=0.999$. This means that, when accepting the null hypothesis $H_{0}$, there is a 0.1 percent probability that the auditor incorrectly accepts the null hypothesis. Vice versa, there is a 99.9 percent probability that the auditor correctly accepts the null hypothesis.

\subsubsection{Comparison of frequentist and Bayesian conclusions}

The $p$ value of 0.468 leads the auditor to not reject the null hypothesis $H_{0}$. Based on this low $p$ value, the auditor cannot say the data contain evidence that supports the conclusion that the auditee's data follow Benford's law. The Bayes factor $B F_{01}=689978$ facilitates the conclusion that the data contain extreme evidence in favor of the conclusion that the auditee's data follow Benford's law.

\subsection{Example 2: Uncovering seasonal patterns}


We now turn to a situation where the auditor uses historical data in an analytical procedure. In particular, the auditor is concerned with the question of how much evidence there is that the sales of the auditee are influenced by seasonal factors. For example, yearly sales revenues may be increasing, but revenues in June might be lower than in September.

As part of the risk assessment process, the auditor can form expectations of patterns that can reasonably be anticipated in the current audit. Often, these expectations involve references to earlier years or industry benchmarks. For example, the auditor may be interested in whether the sales of the auditee are subject to seasonal effects. In addition to a seasonality effect, the auditor wants to know to what extent these historical data support a difference in sales between each season.

\subsubsection{Data}

The data for this example consist of monthly sales of the auditee over the course of the years 2013 - $2016(n=48)$. These data are plotted over time and categorized by season in Figure 2.

\subsubsection{Frequentist analysis}

In the frequentist analysis, the null hypothesis $H_{0}: \mu_{1}=\mu_{2}=\mu_{3}=\mu_{4}$ is assessed via an ANOVA by means of the $p$ value. To get to know more about the specific seasons and to find out if they are significantly different, post-hoc tests are performed and assessed using Tukey's $p$ value, corrected for multiple testing.

Note that we apply ANOVA using a toy model as an illustrative example. In practice, there will be other variables that may also hold predictive value for the sales of the auditee. In all cases, the auditor must carefully construct their statistical model, but can include additional variables using an ANCOVA or a regression analysis. 
We present the results of a frequentist ANOVA testing a main effect for season and post-hoc tests. The results of the ANOVA indicate a significant effect for season $\left(R^{2}=\right.$ $0.3042966, d f=3, F=6.415, p<.01)$. The interpretation of this $p$ value is: assuming that there is no seasonal effect, there is less than one percent probability that the auditor would find the observed (or more extremely deviating) outcomes in the data set. However, as we have shown, the auditor cannot deduce the strength of evidence for the statement that there is a seasonal effect from these $p$ values. Post-hoc tests also indicate a significant difference $(p<$ $.01)$ in the autumn months when compared to the other months, see Table 3. However, when the auditor uses these $p$ values to substantiate this conclusion, they again fall into the aforementioned statistical trap.

\subsubsection{Bayesian analysis}

In a Bayesian analysis, the null model of no effect is compared, by means of the Bayes factor, against an alternative model which incorporates the season as a predictive variable (Rouder et al., 2012; Wetzels, Grasman, \& Wagenmakers, 2012). The post-hoc tests will also be evaluated using the Bayes factor based on the default Bayesian t-test (Rouder et al., 2009; Wetzels et al., 2009). In these post-hoc tests, the posterior odds have been corrected for multiple testing by fixing the prior probability that the null hypothesis holds across all comparisons to 0.5 (Westfall, Johnson, \& Utts, 1997).

The Bayes factor for the model that includes a main effect for season over the model that does not is $B F_{10}=33.923$. This Bayes factor implies that the observed data are 33.923 times more likely to have occurred under the hypothesis of a seasonal effect than under the hypothesis of no seasonal effect, which implies strong evidence for a seasonal effect (see Table 1). To answer the question how much more likely it is that, for example, the autumn season differs from the other seasons, the auditor must inspect the Bayes factors $B F_{10, U}$ 
obtained from the individual comparisons in Table 3. These Bayes factors indicate strong evidence for the statement that the autumn season differs from the spring and winter seasons. However, the data contain only moderate evidence for a difference in the autumn months compared to the winter months.

\subsubsection{Comparison of frequentist and Bayesian conclusions}

The fact that $p<.01$ leads the auditor to reject the null hypothesis $H_{0}$. However, based on this low $p$ value the auditor cannot say there is evidence that supports the conclusion that there is a seasonal effect in the data. In contrast to the $p$ value, the auditor can use the Bayes factor of $B F_{10}=33.924$ to substantiate the conclusion that the data contain strong evidence in favor of a seasonal effect.

\subsection{Example 3: Determining algorithmic bias}

As a final example, we consider an increasingly relevant issue in the context of big data and artificial intelligence. With the rapid growth of information systems that collect and mine customer data, an increasing portion of auditees' business decisions is being guided by artificial intelligence (AI). On 21 April 2021, the European Commission presented a proposal for a regulation concerning artificial intelligence, - the AI Act, for short (European Commission 2021). One major focus of the AI Act is the classification of various types of AI systems according to the risks involved. One of the risks that has special attention is that application of AI might lead to unfair treatment and discrimination. Attention should therefore be given towards ensuring that decisions made with the aid of these algorithms remain fair (Kearns et al., 2018).

For example, such algorithms must avoid exhibiting discriminatory biases towards features such as gender, race, or age. Suppose that the auditor works with an auditee in the banking industry that employs an algorithm to predict whether people are going to default on 
a loan. Naturally, it is highly undesirable that, given that a customer is actually going to pay their loan, they are more likely to get classified by the algorithm as possibly defaulting on that loan as a result of their ethnicity. The following analytical procedure aims to test this algorithmic fairness with respect to ethnicity.

To illustrate this procedure, we focus on a relatively simple criterion of algorithmic fairness. This criterion requires equality of false positive or negative rates across all subgroups in the data (Hardt et al., 2016). In the context of the example algorithm, a false positive would imply that a person gets wrongly marked as a possible defaulter. A false negative on the other hand would mean that a customer will likely default on their loan, but no action will be taken by the bank as this person is not identified by the algorithm. The algorithm may possibly display racial bias if the probability that a customer gets wrongly marked as a possible defaulter is higher for some ethnic groups than for others. Statistically, this implies that the probability of a false positive should be the same across ethnic groups (i.e., the algorithm's classification is independent of a customer's ethnicity). To find out how much evidence there is for this hypothesis, we describe one possible analysis the auditor can use.

\subsubsection{Data}

We use a fictional benchmark data set $(n=10,000)$ from the field of credit risk prediction. The data contain information about a customer's ethnicity, a target variable that indicates defaulting behavior, and other financial information about the customer. Suppose that the auditor has fitted the auditee's predictive model to this benchmark data set to obtain the confusion matrix in Table 4. They calculate the false positive rate $p_{i}$ for each ethnicity $i$ as the number of false positives divided by the number of false positives plus the number of true negatives. 


\subsubsection{Frequentist analysis}

In the frequentist analysis, the null hypothesis of independence $H_{0}: p_{1}=p_{2}=p_{3}=p_{4}$ will be tested using a Chi-squared test and assessed by means of the $p$ value.

The false-positive rates for the categories $p_{1}, p_{2}, p_{3}$ and $p_{4}$ are $0.1051,0.0911,0.1058$, and 0.1033. Using a Chi-square test $\left(X^{2}=3.126, d f=3\right)$, the $p$ value is 0.373 . The interpretation of this $p$ value is: assuming that the false positive rate is equal across all ethnic groups, there is a 37.3 percent probability of observing these (or more extremely deviating) false-positive rates.

\subsubsection{Bayesian analysis}

In the Bayesian analysis, the null hypothesis of independence will be tested against the alternative hypothesis that the false positive rates are dependent on the subgroup (Gunel \& Dickey, 1974; Jamil et al., 2017). The prior distribution for the alternative hypothesis is a $\operatorname{Dirichlet}\left(\alpha_{1}, \alpha_{2}, \alpha_{3}, \alpha_{4}\right)$ distribution with all $\alpha$ parameters set to 1 .

The Bayes factor in favor of $H_{0}$ is $B F_{01}=11077.956$, which implies that the data are 11078 times more likely to have occurred under the hypothesis that the false-positive rates are equal across ethnic groups than under the hypothesis that they are not. Using this Bayes factor, the auditor can quantify evidence in favor of the null hypothesis and support the statement that the false positive rates are equal across groups.

\subsubsection{Comparison of frequentist and Bayesian conclusions}

The $p$ value of 0.373 leads the auditor to not reject the null hypothesis $H_{0}$. However, based on this $p$ value, the auditor cannot say the data shows evidence that supports the alternative hypothesis: the false-positive rates are equal across ethnicities. In contrast to the $p$ 
value, the auditor can use the Bayes factor of $B F_{01}=11077.956$ to substantiate the conclusion that the data contain extreme evidence in favor of equal false-positive rates across ethnicities.

\section{CONCLUDING COMMENTS}

From the perspective of an auditor, audit evidence plays a crucial role in providing an opinion about whether the assertions presented by the auditee's management in the financial statements are credible. However, the frequentist method (NHST) by which statistical audit evidence is currently often quantified in audits has raised legitimate concerns over the years. In this article, we have emphasized the fact that a frequentist hypothesis test does not produce the type of evidence that the audit standards advocate, and that the $p$ value does not fit well with the nature of audit questions. We have shown that a Bayesian hypothesis test can produce, in certain situations, a more fitting type of evidence for the auditor's conclusions about the financial statements, and that it does not suffer from the same limitations as the $p$ value when it comes to effectiveness and efficiency. Because the Bayes factor can quantify evidence in both directions, the Bayesian approach to audit evidence is more in line with the audit standards than that of a frequentist hypothesis test. We therefore propose the Bayes factor as an addition to the auditor's statistical toolbox. Since the auditing standards explicitly call for evidence that can support or contradict the auditor's conclusions, we expect that the Bayes factor will enhance the way that auditors are able to quantify and evaluate statistical evidence from a sample.

Moreover, Bayesian inference provides auditors the tools to aggregate audit evidence, and therefore to statistically accumulate audit evidence over the course of an audit. This makes the Bayes factor a good fit for today's audit practice because it can facilitate the growing use of complex data analytics by the auditor and the auditee. As data will become 
more complex, and statistical analyses will become more prevalent, the auditor will require an intuitive framework to integrate, quantify, and interpret the information from these procedures. This will be especially the case if they are to meet the constant demand for a more efficient audit. Since the Bayesian framework provides the flexibility to incorporate many types of prior information into the statistical analysis, we believe that it will be more useful for the auditor in the long term than the current frequentist methods.

Despite our arguments in favor of Bayesian hypothesis testing using the Bayes factor, it is not always practical to use this approach in favor of a frequentist hypothesis test. As discussed in Section 2, Bayes' theorem utilizes prior information in the form of the prior probabilities and the prior distribution on the parameters to perform inference. This means that, to get to the Bayes factor in practice, the auditor will have to think about how they incorporate their existing information into the statistical model. This has as a consequence that sometimes, a frequentist hypothesis test can be more beneficial to an auditor if translating prior information into Bayesian prior distributions is difficult, expensive or time-consuming. Although the Bayesian approach comes with advantages such as being able to quantify evidence in favor and against the auditor's conclusions or the ability to engage in sequential testing without penalty, the auditor needs to decide whether they think that the benefits of the Bayesian approach outweigh the costs of justifying that approach.

The sensitivity of the Bayes factor to the prior distribution is an avenue for further research in this area. For Bayesian hypothesis testing, default prior distributions exist and have been evaluated in a wide variety of designs and settings (Rouder et al., 2009; Wetzels \& Wagenmakers, 2012; Wetzels, Grasman, \& Wagenmakers, 2012). However, no default prior distribution exists or has been evaluated specifically in the context of audit sampling. Moreover, it remains to be investigated how auditors use and interpret Bayesian evidence in practice, and if it increases the ease of interpretation of statistical results for auditors. 
The examples shown in this manuscript show a selection of data-rich audit scenarios that the Bayes factor can be applied in, but in principle any statistical analysis can be performed in a Bayesian fashion. Most Bayesian analyses are easily accessible in a standardized format through open-source software packages (e.g., R) or graphical user interfaces (e.g., JASP). We have performed all statistical analyses in this manuscript using JASP (JASP Team, 2021) and have included reproducible examples in the online appendix to this article. Our proposition for a way forward is that next to their frequentist analyses auditors perform Bayesian equivalents of these analyses to become acquainted with these techniques, and to be able to compare the two measures of evidence ( $p$ values and Bayes factors) in practice.

To conclude, we suggest the use of Bayesian inference in the audit because it fits well with the goals of the auditor. First, the Bayes factor embodies the audit standards' description of audit evidence and provides the auditor with a measure of statistical evidence that can support or contradict their conclusions. Second, the theoretical foundations underlying the Bayesian framework have long been argued to be beneficial for the audit, since they enable the auditor to quantify and aggregate evidence over the audit using the prior and posterior probabilities. In sum, Bayesian inference provides a fitting answer to the problems that today's auditors face. 


\section{REFERENCES}

Altman, D. G., \& Bland, J. M. (1995). Absence of evidence is not evidence of absence. British Medical Journal, 311, 485.

American Institute of Certified Public Accountants (AICPA). (2019). Audit Sampling: Audit Guide. New York, NY: AICPA.

American Institute of Certified Public Accountants (AICPA). (2021). Clarified Statements on Auditing Standards. New York, NY: AICPA.

Appelbaum, D. A., Kogan, A., \& Vasarhelyi, M. A. (2018). Analytical procedures in external auditing: A comprehensive literature survey and framework for external audit analytics. Journal of Accounting Literature, 40, 83-101.

Armitage, P., McPherson, C. K., \& Rowe, B. C. (1969). Repeated significance tests on accumulating data. Journal of the Royal Statistical Society: Series A (General), 132(2), $235-244$.

van Batenburg, P. 2018. Uitbreiden van steekproeven om beter te schatten. In Kloosterman, H., van Batenburg, P., and Voorhoeve, K. (Ed.). Essaybundel Statistical Auditing (pp. 119 - 121), 1st edition. Publishing House Jacques de Swart.

Beck, P. J., Solomon, I., \& Tomassini, L. A. (1985). Subjective prior probability distributions and audit risk. Journal of Accounting Research, 23(1), 37-56.

Benford, F. (1938). The law of anomalous numbers. In Proceedings of the American Philosophical Society, 551-572.

Bennett, G. B., \& Hatfield, R. C. (2013). The effect of the social mismatch between staff auditors and client management on the collection of audit evidence. The Accounting Review, 88(1), 31-50. 
Berger, J. O., \& Sellke, T. (1987). Testing a point null hypothesis: The irreconcilability of $p$ values and evidence. Journal of the American Statistical Association, 82(397), 112-122.

Berger, J. O., \& Wolpert, R. L. 1988. The likelihood principle (2nd ed.). Hayward, CA: Institute of Mathematical Statistics.

Berkson, J. (1942). Tests of significance considered as evidence. Journal of the American Statistical Association, 37(219), 325-335.

Bollen, K. A., Ray, S., Zavisca, J., \& Harden, J. J. (2012). A comparison of Bayes factor approximation methods including two new methods. Sociological Methods \& Research, 41(2), 294-324.

Cipriani, M., Costantini, R., \& Guarino, A. (2012). A Bayesian approach to experimental analysis: Trading in a laboratory financial market. Review of Economic Design, 16(2), $175-191$.

Corless, J. C. (1972). Assessing prior distributions for applying Bayesian statistics in auditing. The Accounting Review, 47(3), 556-566.

Daroca, F. P., \& Holder, W. W. (1985). The use of analytical procedures in review and audit engagements. Auditing: A Journal of Practice and Theory, 4(2), 80-92.

Derks, K., de Swart, J., van Batenburg, P., Wagenmakers, E.-J., \& Wetzels, R. (2021). Priors in a Bayesian audit: How integration of existing information into the prior distribution can improve audit transparency and efficiency. International Journal of Auditing, 1-16.

van Doorn J, van den Bergh, D., Bohm, U., Dablander, F., Derks, K., Draws, T., Evans, N. J., Gronau, Q. F., Hinne, M., Kucharský, Š., Ly, A., Marsman, M., Matzke, D., Raj, A., Sarafoglou, A., Stefan, A., Voelkel, J. G., \& Wagenmakers, E.-J. (2020). The JASP 
guidelines for conducting and reporting a Bayesian analysis. Psychonomic Bulletin and Review, 1-14.

Dowling, C., \& Leech, S. (2007). Audit support systems and decision aids: Current practice and opportunities for future research. International Journal of Accounting Information Systems, 8(2), 92-116.

Drake, P. D., \& Nigrini, M. J. (2000). Computer assisted analytical procedures using Benford's Law. Journal of Accounting Education, 18(2), 127-146.

Durtschi, C., Hillison, W., \& Pacini, C. (2004). The effective use of Benford's law to assist in detecting fraud in accounting data. Journal of forensic accounting, 5(1), 17-34.

Edmonds, M., Miller, T., \& Savage, A. (2019). Accounts receivable: An audit simulation. Journal of Accounting Education, 47, 75-92.

Edwards, W., Lindman, H., \& Savage, L. J. (1963). Bayesian statistical inference for psychological research. Psychological Review, 70(3), 193-242.

Elliott, R. K., \& Rogers, J. R. (1972). Relating statistical sampling to audit objectives. Journal of Accountancy, 134, 46-55.

European Commission. 2021. Proposal for a Regulation of the European Parliament and of the Council laying down harmonised rules on artificial intelligence (Artificial Intelligence Act) and amending certain Union legislative acts, https://eurlex.europa.eu/legal-content/EN/TXT/?uri=CELEX\%3A52021PC0206

Fisher, R. A. (1934). Statistical methods for research workers (5th ed.). London: Oliver \& Boyd.

Gillett, P. R., \& Srivastava, R. P. (2000). Attribute Sampling: A Belief-Function Approach to Statistical Audit Evidence. Auditing: A Journal of Practice \& Theory, 19(1), 145-155. 
Good, I. J. (1967). A Bayesian significance test for multinomial distributions. Journal of the Royal Statistical Society - Series B (Methodological), 29, 399-431.

Goodman, S. (2008). A dirty dozen: Twelve p-value misconceptions. Seminars in Hematology, 45, 135-140.

Goodman, S. N., \& Royall, R. (1988). Evidence and scientific research. American Journal of Public Health, 78(12), 1568-1574.

Gunel, E. \& Dickey, J. (1974). Bayes factors for independence in contingency tables. Biometrika, 61(3), 545-557. https://doi.org/10.2307/2334738

Heck, D. W., Boehm, U., Böing-Messing, F., Bürkner, P., Derks, K., Dienes, Z., Fu, Q., Gu, X., Karimova, D., Kiers, H. A. L., Klugkist, I., Kuiper, R. M., Lee, M. D., Leenders, R., Leplaa, H. J., Linde, M., Ly, A., Meijerink-Bosman, M., Moerbeek, M., Mulder, J., Palfi, B., Schönbrodt, F. D., Tendeiro, J. N., van den Bergh, D., van Lissa, C., van Ravenzwaaij, D., Vanpaemel, W., Wagenmakers, E.-J., Williams, D. R., ZondervanZwijnenburg, M., \& Hoijtink, H. (2020). A review of applications of the Bayes factor in psychological research. PsyArXiv. Retrieved from https://doi.org/10.31234/osf.io/cu43g

Hubbard, R., \& Lindsay, R. M. (2008). Why P values are not a useful measure of evidence in statistical significance testing. Theory \& Psychology, 18(1), 69-88.

International Auditing and Assurance Standards Board (IAASB). (2018). Handbook of International Quality Control, Auditing Review, Other Assurance, and Related Services Pronouncements, part I. New York: International Federation of Accountants.

Jamil, T., Ly, A., Morey, R. D., Love, J., Marsman, M., \& Wagenmakers, E.-J. (2017). Default "Gunel and Dickey" Bayes factors for contingency tables. Behavior Research Methods, 49(2), 638-652. 
JASP Team. (2022). JASP (Version 0.16.2)[Computer software].

Jeffreys, H. (1939). Theory of Probability (1st ed.). Oxford University Press. Oxford.

Johnstone, D. J. (1986). Tests of significance in theory and practice. Journal of the Royal Statistical Society: Series D (The Statistician), 35(5), 491-498.

Johnstone, D. J. (1990). Sample size and the strength of evidence: A Bayesian interpretation of binomial tests of the information content of qualified audit reports. Abacus, 26(1), $17-35$.

Johnstone, D. J. (1994). A Statistical Paradox in Auditing. Abacus, 30(1), 44-49.

Johnstone, D. (2018). Accounting theory as a Bayesian discipline. Foundations and Trends in Accounting, 13(1-2), 1-266.

Johnstone, D. (2021). Accounting research and the significance test crisis. Critical Perspectives on Accounting, 54(4): 524-546.

Kass, R. E., \& Raftery, A. E. (1995). Bayes factors. Journal of the American Statistical Association, 90(430), 773-795.

Keysers, C., Gazzola, V., \& Wagenmakers, E.-J. (2020). Using Bayes factor hypothesis testing in neuroscience to establish evidence of absence. Nature Neuroscience, 23(7), 788-799.

Kim, J. H., Ahmed, K., \& Ji, P. I. (2018). Significance testing in accounting research: A critical evaluation based on evidence. Abacus, 54(4), 524-546.

Kinney, W. R. (1975). Decision theory aspects of internal control system design/compliance and substantive tests. Journal of Accounting Research, 13, 14-29. 
Lehmann, E. L., \& Romano, J. P. (2006). Testing statistical hypotheses. Springer Science \& Business Media.

Leslie, D. A. (1984). Analysis of the audit framework focusing on inherent risk and the role of statistical sampling in compliance testing. In Proceedings of the 1984 Touche Ross University of Kansas Symposium of Auditing Problems, eds. H. Stettler, and N. Ford, 89-125. Lawrence, KA: School of Business, University of Kansas.

Li, C., Raman, K. K., Sun, L., \& Yang, R. (2020). The SOX 404 control audit and the effectiveness of additional audit effort in lowering the risk of financial misstatements. Review of Quantitative Finance and Accounting, 54(3), 981-1009.

Lindley, D. V. (1993). The analysis of experimental data: The appreciation of tea and wine. Teaching Statistics, 15, 22-25.

Love, J., Selker, R., Marsman, M., Jamil, T., Dropmann, D., Verhagen, J., Ly, A., Gronau, Q. F., Smira, M., Epskamp, S., Matzke, D., Wild, A., Knight, P., Rouder, J. N., Morey, R. D., \& Wagenmakers, E.-J. (2019). JASP: Graphical statistical software for common statistical designs. Journal of Statistical Software, 88(2), 1-17.

Ly, A., Raj, A., Etz, A., Marsman, M., Gronau, Q. F., \& Wagenmakers, E.-J. (2018). Bayesian reanalyses from summary statistics: A guide for academic consumers. Advances in Methods and Practices in Psychological Science, 1(3), 367-374.

Ly, A., Verhagen, J., \& Wagenmakers, E.-J. (2016). Harold Jeffreys's default Bayes factor hypothesis tests: Explanation, extension, and application in psychology. Journal of Mathematical Psychology, 72, 19-32.

Lynch, S. M., \& Bartlett, B. (2019). Bayesian statistics in sociology: Past, present, and future. Annual Review of Sociology, 45, 47-68. 
Ma'arif, M. Mohd Satar, N., Abdul Jalal, A. A., \& Samah, M. (2020). Detecting erp data fraud using the first digits formula of Benford's law. Science International, 32(4), 439-444.

Martel-Escobar, M., Vázquez-Polo, F. J., \& Hernández-Bastida, A. (2018). Bayesian inference in auditing with partial prior information using maximum entropy priors. Entropy, 20, 919.

Nigrini, M. J. (2012). Benford's Law: Applications for forensic accounting, auditing, and fraud detection (Vol. 586). John Wiley \& Sons.

Nigrini, M. J., \& Mittermaier, L. J. (1997). The use of Benford's law as an aid in analytical procedures. Auditing, 16(2), 52-67.

Perry, T. P. M. (2011). SAS 106-Audit Evidence: Is it Effective?. Available at SSRN 1907438.

Public Company Accounting Oversight Board (PCAOB). (2020). Auditing Standards. Washington, WA: PCAOB.

Richard, M., \& Vecer, J. (2021). Efficiency testing of prediction markets: Martingale approach, likelihood ratio and Bayes factor analysis. Risks, 9(2), 31. DOI: https://doi.org/10.3390/risks9020031

Roberts, D. M. (1975). Discussion of The Real Risks in Audit Sampling. Journal of Accounting Research, 13, 92-94. https://doi.org/10.2307/2490481

Rose, A. M., Rose, J. M., Suh, I., \& Thibodeau, J. C. (2020). Analytical procedures: Are more good ideas always better for audit quality?. Behavioral Research in Accounting, 32(1), 37-49.

Rouder, J. N. (2014). Optional stopping: No problem for Bayesians. Psychonomic bulletin \& review, 21(2), 301-308. 
Rouder, J. N., Morey, R. D., Speckman, P. L., \& Province, J. M. (2012). Default Bayes factors for ANOVA designs. Journal of Mathematical Psychology, 56(5), 356-374. https://doi.org/10.1016/j.jmp.2012.08.001

Rouder, J. N., Speckman, P. L., Sun, D., Morey, R. D., \& Iverson, G. (2009). Bayesian t-tests for accepting and rejecting the null hypothesis. Psychonomic Bulletin \& Review, 16, $225-237$.

Sarafoglou, A., Haaf, J. M., Ly, A., Gronau, Q. F., \& Wagenmakers, E.-J. (2020). Evaluating multinomial order restrictions with bridge sampling. PsyArXiv. Retrieved from https://doi.org/10.31234/osf.io/bux7p.

Scott, W. R. (1973). A Bayesian approach to asset valuation and audit size. Journal of Accounting Research, 11(2), 304-330.

Sorensen, J. E. (1969). Bayesian analysis in auditing. The Accounting Review, 44(3), 555-561. Steele, A. (1992). Audit risk and audit evidence: The Bayesian approach to statistical auditing. London: Academic Press.

Stewart, T. R. (2012). Technical notes on the AICPA audit guide Audit Sampling. American Institute of Certified Public Accountants. New York: 5-8.

Stewart, T. R. (2013). A Bayesian audit assurance model with application to the component materiality problem in group audits. VU University. Amsterdam.

Van den Acker, C. (2000). Belief-function representation of statistical audit evidence. International journal of intelligent systems, 15(4), 277-290.

Varma, D. T., \& Khan, D. A. (2012). Fraud detection in supply chain using Benford distribution. International Journal of Research in Management, 5(2). 
Wagenmakers, E.-J. (2007). A practical solution to the pervasive problems of $p$ values. Psychonomic bulletin \& review, 14(5), 779-804.

Wagenmakers, E., Gronau, Q. F., \& Vandekerckhove, J. (2019). Five Bayesian intuitions for the stopping rule principle. PsyArXiv. Retrieved from https://doi.org/10.31234/osf.io/5ntkd

Wagenmakers, E.-J., Lee, M., Lodewyckx, T., \& Iverson, G. J. (2008). Bayesian versus frequentist inference. In Bayesian evaluation of informative hypotheses (pp. 181-207). Springer, New York, NY.

Wasserstein, R. L., \& Lazar, N. A. (2016). The ASA statement on p-values: context, process, and purpose. The American Statistician, 70(2), 129-133.

Wei, A. \& Vellwock, A. E. (2020). Is COVID-19 data reliable? A statistical analysis with Benford's law. DOI: 10.13140/RG.2.2.31321.75365/1

Westfall, P. H., Johnson, W. O., \& Utts, J. M. (1997). A Bayesian perspective on the Bonferroni adjustment. Biometrika, 84(2), 419-427.

Wetzels, R., Grasman, R. P., \& Wagenmakers, E.-J. (2012). A default Bayesian hypothesis test for ANOVA designs. The American Statistician, 66(2), 104-111.

Wetzels, R., Matzke, D., Lee, M. D., Rouder, J. N., Iverson, G. J., \& Wagenmakers, E.-J. (2011). Statistical evidence in experimental psychology: An empirical comparison using 855 t tests. Perspectives on Psychological Science, 6(3), 291-298.

Wetzels, R., Raaijmakers, J. G., Jakab, E., \& Wagenmakers, E.-J. 2009. How to quantify support for and against the null hypothesis: A flexible WinBUGS implementation of a default Bayesian $t$ test. Psychonomic Bulletin \& Review, 16, 752-760. 
Wetzels, R., \& Wagenmakers, E.-J. (2012). A default Bayesian hypothesis test for correlations and partial correlations. Psychonomic Bulletin \& Review, 19(6), 1057-1064.

Yin, X. (2020). Audit Evidence Concept, Classification and Collection Techniques in China and the US. Global Journal of Management and Business Research, 19(3), 1-6. 


\section{APPENDIX - TWO APPROACHES TO NHST IN AUDIT SAMPLING}

There are two approaches to frequentist null hypothesis significance testing (NHST) in audit sampling: the positive approach and the negative approach (Roberts, 1975). The key difference between the positive and the negative approach comes down to how the hypothesis of tolerable misstatement is defined, that is, whether the hypothesis of tolerable misstatement includes the critical error limit (i.e., the performance materiality) or not. It can be demonstrated that the two approaches are equivalent (Roberts, 1978, p. 45).

In the positive approach, the value of the performance materiality is seen as the maximum tolerable misstatement. Hence, in this approach the hypothesis of tolerable misstatement includes the value of the performance materiality. This null hypothesis is encapsulated by the statistical scenario where $\theta \leq 0.03$, whereas the alternative hypothesis of intolerable misstatement is encapsulated by the scenario where $\theta>0.03$. Because in NHST the auditor assumes that the null hypothesis is true, the positive approach implies a philosophy of auditing where the auditor accepts the population as not materially misstated unless there is evidence to the contrary. The sampling risks $\alpha$ and $\beta$ are those defined in the main article: $\alpha$ is the risk of incorrectly deciding that the population contains material misstatement when in fact it does not (i.e., a Type-I error) and $\beta$ is the risk of incorrectly deciding that the population does not contain material misstatement when in fact it does (i.e., a Type-II error).

In the negative approach, the value of the performance materiality is seen as the minimum intolerable misstatement. Hence, in this approach the hypothesis of tolerable misstatement does not include the value of the performance materiality. This alternative hypothesis is encapsulated by the statistical scenario where $\theta<0.03$, whereas the null hypothesis of intolerable misstatement is encapsulated by the scenario where $\theta \geq 0.03$. Because in NHST the auditor assumes that the null hypothesis is true, the negative approach implies a philosophy of auditing where the auditor rejects the population as being materially 
misstated unless there is evidence to the contrary. As is the case for the null and alternative hypotheses, the interpretation of the sampling risks $\alpha$ and $\beta$ is reversed in this approach: $\alpha$ is the risk of incorrectly deciding that the population does not contain material misstatement when in fact it does (i.e., a Type-I error) and $\beta$ is the risk of incorrectly deciding that the population contains material misstatement when in fact it does not (i.e., a Type-II error).

In the article accompanying this appendix, we have focused on the positive approach to NHST in audit sampling for two reasons. First, the text of the auditing standards directly links to the positive approach. For example, the definition of performance materiality is given as: "Performance materiality is determined to reduce to an appropriately low level the probability that the aggregate of uncorrected and undetected misstatements in the financial statements exceeds materiality for the financial statements as a whole" (ISA 320, paragraph A.13, International Auditing and Assurance Board (IAASB), 2018). Furthermore, the definition of tolerable misstatement (i.e., the application of performance materiality to a particular sampling procedure) is given as: "A monetary amount set by the auditor in respect of which the auditor seeks to obtain an appropriate level of assurance that the monetary amount set by the auditor is not exceeded by the actual misstatement in the population" (ISA 530, paragraph 5i, International Auditing and Assurance Board (IAASB), 2018, AU-C 530, paragraph 5, American Institute of Certified Public Accountants (AICPA), 2021). These texts highlight the intention of the auditor to assert whether the actual misstatement (i.e., $\theta$ ) does not exceed (i.e., $\leq$ ) the performance materiality or tolerable misstatement (i.e., 0.03). The fact that $\theta$ must not exceed the performance materiality implies that the value of the performance materiality is included in the hypothesis of tolerable misstatement (i.e., $\theta \leq 0.03$ ). A second argument for the positive approach is that this approach is prominent in the auditing literature, see for example Elliott \& Rogers (1972), Johnstone (1994), Martel-Escobar et al. (2018), and Edmonds et al., (2019). 
However, there are also several arguments to be made in favor of the negative approach. First, in hypothesis testing, the probability of incorrectly rejecting the null hypothesis is generally considered to be more important and is therefore referred to as primary risk, Type-I error, $\alpha$, or audit risk. This preference for the $\alpha$ risk as the conceptualization of audit risk is consistent with the negative approach described above. In an audit context, the negative approach can be seen as a form of "guilty-until-proven-innocent", where "guilty" means: the financial statements contain material misstatement. Thus, in the negative approach the auditor audits the financial statements from a conservative point of view. A second argument for the negative approach is that the standard sample size tables in AICPA (2019, appendix A and appendix C) can only be replicated using this approach. Hence, because practitioners may be more familiar with the negative approach to NHST, we describe the calculations underlying the running example from the article in the remainder of this appendix.

To determine when $H_{0}: \theta \geq 0.03$ should be rejected for a given sample size, the auditor must calculate the maximum number of incorrect signatures that can be observed while the risk of incorrectly rejecting the null hypothesis is still sufficiently low. Suppose that the auditor has determined $\alpha$-the risk of incorrectly deciding that the population does not contain material misstatement when in fact it does- to be five percent. In this case, the rule for rejection of $H_{0}$ is $k=0$ because if it is true that $\theta=0.03$, then the probability of finding 0 incorrect signatures in the sample of 99 items equals 4.99\% (see Equation A.1). Using a higher threshold, as in $k=1$, would violate this sampling risk since the probability of finding 1 or less incorrect signatures is $19.91 \%$. As described in the manuscript, this procedure for rejecting $H_{0}$ can also be regarded as rejecting the null hypothesis if the $p$ value associated with the observed value of $k$ is less than or equal to the significance level $\alpha$. 
Let's revisit the running example in which a sample of 99 purchase orders was selected and no incorrect signatures were found. In this case, the $p$ value is the probability of finding $k=0$ incorrect signatures in the sample, given that the purchase orders contain three percent misstatement, and equals $p=0.049$ (Equation A.1). Since the calculated $p$ value of 0.049 is lower than the significance level $\alpha=0.05$, the auditor can reject the null hypothesis.

$$
p=\left(\begin{array}{c}
99 \\
0
\end{array}\right) 0.03^{0}(1-0.03)^{99-0}=0.049
$$

The auditor can also calculate the sampling risk $\beta$-the risk of deciding that the population contains material misstatement when in fact it does not. Like in the positive approach, to calculate $\beta$ for an alternative point hypothesis about the population misstatement, the auditor needs to make an assumption about $\theta$. Suppose the auditor assumes that the population misstatement is equal to $\theta=0.02$, which is slightly lower than the performance materiality. When assuming the truth of this hypothesis, the risk of failing to reject the null hypothesis $H_{0}: \theta \geq 0.03$ is the probability of finding an outcome that would yield a $p$ value above five percent (e.g., $k=1$ misstatements would give $p>.05$ and thus lead the auditor to not reject $H_{0}$ ). Hence, the sampling risk $\beta$ can be calculated as the probability of finding $k=1$ to $k=99$ incorrect signatures in the sample under the $\operatorname{binomial}(k \mid n=99, \theta=0.02)$ distribution and equals $\beta=0.86$.

From a Bayesian point of view, the two hypotheses $H_{0}: \theta \geq 0.03$ and $H_{1}: \theta<0.03$ are defined as the range of the prior distribution that corresponds to the hypotheses' restrictions with respect to $\theta$. This means that the prior probability for the hypothesis $H_{0}$ corresponds to the total probability under the prior distribution on $\theta$ in the range $(0.03 ; 1]$. Vice versa, the prior probability for the hypothesis $H_{1}$ corresponds to the total probability under the prior distribution on $\theta$ in the range [0;0.03]. Like in the manuscript, the auditor chooses to specify a beta $(0,34)$ prior distribution for $\theta$. 
After being updated by the sample of $n=99$ items of which $k=0$ contains an error, the posterior distribution is the beta $(1+0=1,34+99=133)$ distribution. The posterior odds in favor of $H_{1}$ induced by the posterior distribution are therefore $\frac{0.983}{0.017}=57.824$. The posterior probability $\mathrm{p}\left(H_{1} \mid y\right)=0.983$ implies that there is a 98.3 percent probability that the population does not contain misstatements equal to or above the performance materiality. This means that, when accepting $H_{1}$, there is a 1.7 percent probability that the auditor incorrectly judges that the population is free of material misstatement. This probability is sufficiently low to find the statement in the alternative hypothesis credible. Vice versa, this also implies that there is a 98.3 percent probability that the auditor correctly judges that the population is not materially misstated.

Because we know the prior odds and the posterior odds, we can again calculate the Bayes factor by dividing the two: $B F_{10}=\frac{57.824}{1.817} \approx 31$. This Bayes factor implies that the data are about 31 times more likely to occur under $H_{1}$ than under $H_{0}$. 
Table 1. Bayes factor labels as proposed by Jeffreys (1961).

$$
B F_{01}=\frac{1}{B F_{10}} \quad \text { Strength of evidence }
$$

$<\frac{1}{100} \quad$ Extreme evidence for $H_{1}$

$\frac{1}{100}-\frac{1}{30}$ Very strong evidence for $H_{1}$

$\frac{1}{30}-\frac{1}{10} \quad$ Strong evidence for $H_{1}$

$\frac{1}{10}-\frac{1}{3}$ Moderate evidence for $H_{1}$

$\frac{1}{3}-1 \quad$ Anecdotal evidence for $H_{1}$

1 No evidence for $H_{0}$ or $H_{1}$

1 - 3 Anecdotal evidence for $H_{0}$

3 - 10 Moderate evidence for $H_{0}$

10 - 30 Strong evidence for $H_{0}$

$30-100$ Very strong evidence for $H_{0}$

$>100$ Extreme evidence for $H_{0}$ 
Table 2. Descriptive statistics for the first digits in the Sino Forest data set.

\begin{tabular}{|c|c|c|c|}
\hline Leading digit & Count & Percentage & Benford's law \\
\hline 1 & 231 & $29.92 \%$ & $30.1 \%$ \\
\hline 2 & 124 & $16.06 \%$ & $17.61 \%$ \\
\hline 3 & 97 & $12.56 \%$ & $12.49 \%$ \\
\hline 4 & 70 & $9.07 \%$ & $9.69 \%$ \\
\hline 5 & 64 & $8.29 \%$ & $7.92 \%$ \\
\hline 6 & 54 & $6.99 \%$ & $6.69 \%$ \\
\hline 7 & 40 & $5.18 \%$ & $5.8 \%$ \\
\hline 8 & 54 & $6.99 \%$ & $5.12 \%$ \\
\hline 9 & 38 & $4.92 \%$ & $4.58 \%$ \\
\hline
\end{tabular}


Table 3. ANOVA post-hoc comparisons for season.

\begin{tabular}{|c|c|c|c|c|c|c|c|c|}
\hline & & Mean Difference & SE & $\mathrm{t}$ & $\mathrm{p}_{\text {tukey }}$ & Prior Odds & Posterior Odds & $\mathrm{BF}_{10, \mathrm{U}}$ \\
\hline \multirow[t]{3}{*}{ Autumn } & Spring & 30168.261 & 8876.126 & 3.399 & 0.008 & 0.414 & 16.053 & 38.755 \\
\hline & Summer & 33339.340 & 8876.126 & 3.756 & 0.003 & 0.414 & 41.542 & 100.291 \\
\hline & Winter & 31554.932 & 8876.126 & 3.555 & 0.005 & 0.414 & 1.775 & 4.285 \\
\hline \multirow[t]{2}{*}{ Spring } & Summer & 3171.079 & 8876.126 & 0.357 & 0.984 & 0.414 & 0.183 & 0.442 \\
\hline & Winter & 1386.672 & 8876.126 & 0.156 & 0.999 & 0.414 & 0.156 & 0.376 \\
\hline Summer & Winter & -1784.408 & 8876.126 & -0.201 & 0.997 & 0.414 & 0.156 & 0.378 \\
\hline
\end{tabular}

Note. P value adjusted for comparing a family of 4 . The posterior odds have been corrected for multiple testing by fixing to 0.5 the prior probability that the null hypothesis holds across all comparisons (Westfall, Johnson, and Utts, 1997). Individual comparisons are based on the default t-test with a Cauchy $(0, r=1 / s q r t(2))$ prior. The "U" in the Bayes factor denotes that it is uncorrected. 
Table 4. The confusion matrix obtained from the auditee's classification algorithm.

\begin{tabular}{|c|c|c|c|c|}
\hline \multirow[b]{2}{*}{ Ethnicity } & \multirow[b]{2}{*}{ Observed } & \multicolumn{2}{|c|}{ Predicted } & \multirow[b]{2}{*}{ Total } \\
\hline & & Default & Paid & \\
\hline \multirow[t]{3}{*}{ Asian } & Paid & 97 & 826 & 923 \\
\hline & Defaulted & 11 & 66 & 77 \\
\hline & Total & 108 & 892 & 1000 \\
\hline \multirow[t]{3}{*}{ African } & Paid & 167 & 1678 & 1845 \\
\hline & Defaulted & 12 & 143 & 155 \\
\hline & Total & 179 & 1821 & 2000 \\
\hline \multirow[t]{3}{*}{ Hispanic } & Paid & 195 & 1648 & 1843 \\
\hline & Defaulted & 17 & 140 & 157 \\
\hline & Total & 212 & 1788 & 2000 \\
\hline \multirow[t]{3}{*}{ Caucasian } & Paid & 477 & 4137 & 4614 \\
\hline & Defaulted & 55 & 331 & 386 \\
\hline & Total & 532 & 4468 & 5000 \\
\hline \multirow[t]{3}{*}{ Total } & Paid & 936 & 8289 & 9225 \\
\hline & Defaulted & 95 & 680 & 775 \\
\hline & Total & 1031 & 8969 & 10000 \\
\hline
\end{tabular}



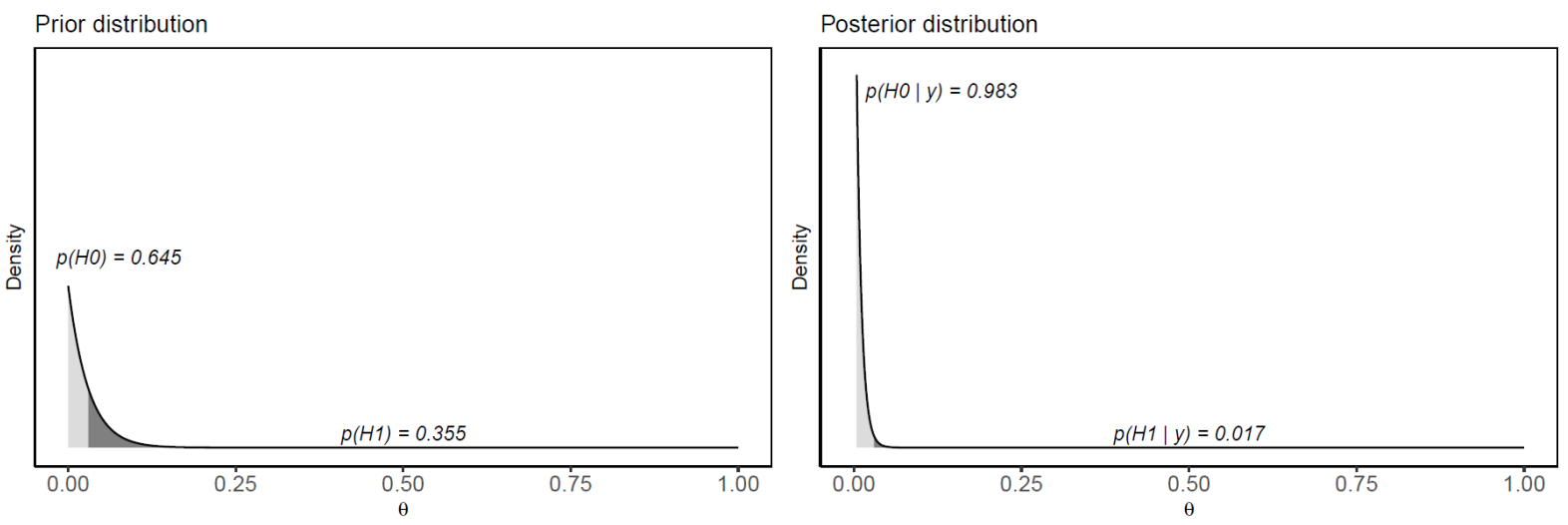

Figure 1. The beta $(1,34)$ prior distribution (left panel) and beta(1, 133) posterior distribution (right panel) on the misstatement proportion $\theta$ after seeing a sample of $n=99$ items containing $k=0$ misstatements. The prior-and posterior probabilities for $H_{0}$ (light) and $H_{1}$ (dark) induced by the prior-and posterior distributions are shown in numbers. 

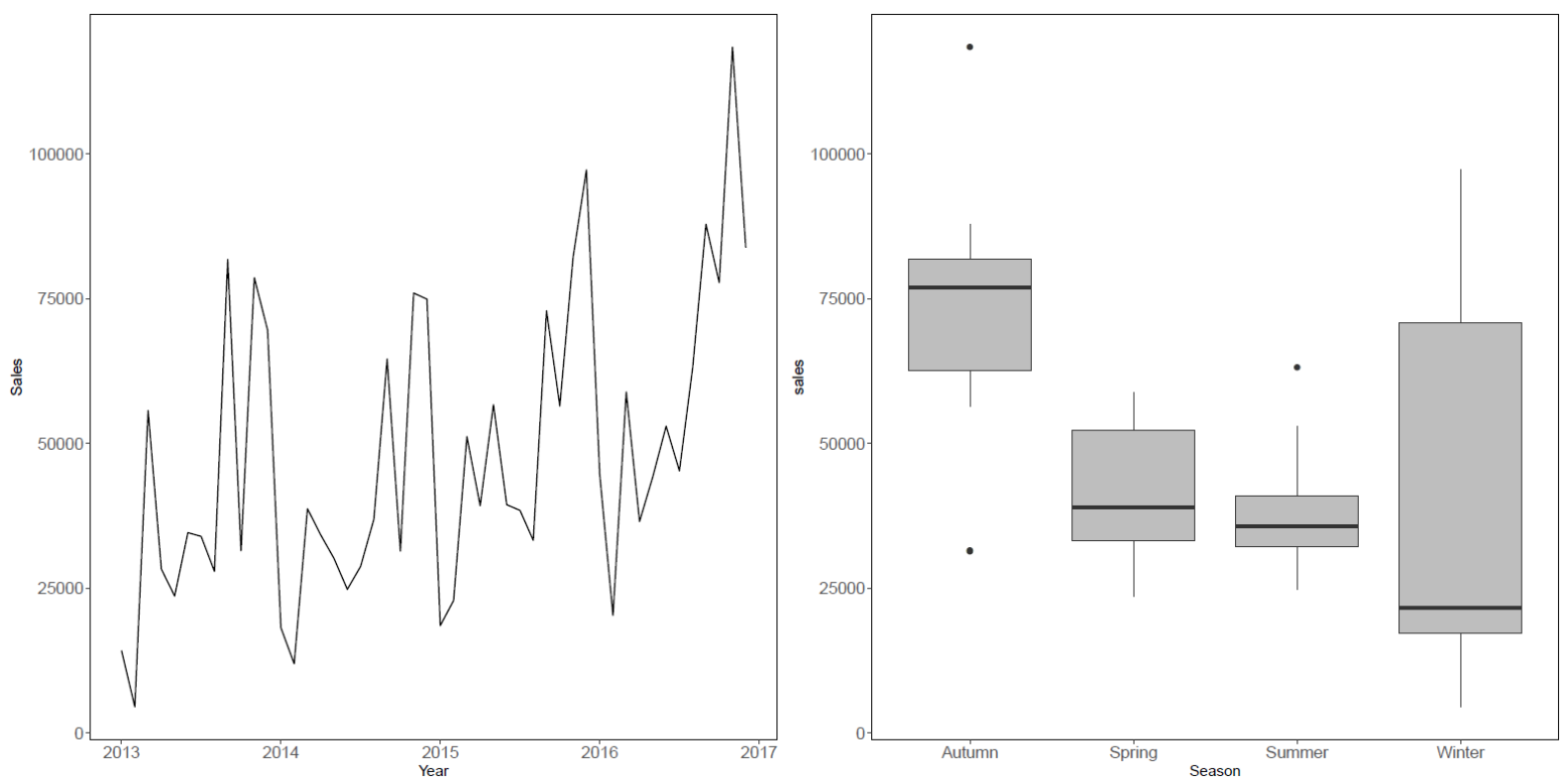

Figure 2. Monthly sales of the auditee over the course of the years 2013 - 2016. The left panel shows the sales over time, and the right panel shows the sales categorized by season. 